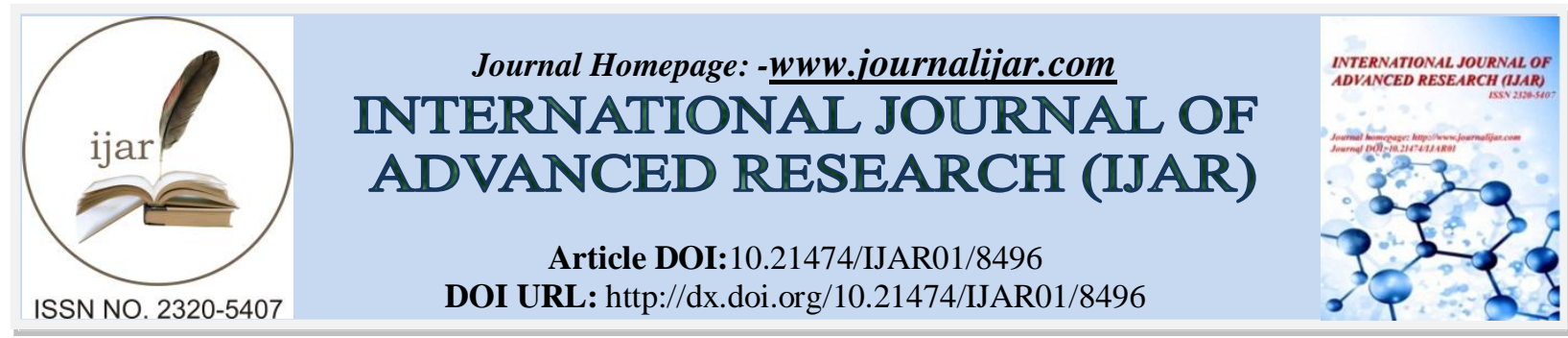

RESEARCH ARTICLE

\title{
OPTIMIZATION OF REVERSE-AODV ON EMERGENCY BROADCAST SIMULATION.
}

\section{Akbar Marwan, Ruddy J Suhatril and Rahmat Budi Wicaksono.}

Faculty of Industrial Technology, Gunadarma University.

\section{Manuscript Info}

Manuscript History

Received: 04 December 2018

Final Accepted: 06 January 2019

Published: February 2019

Key words:-

RAODV, Broadcast Simulation,

Wireless.

\begin{abstract}
In addition to the use of wire as a delivery media, currently there is an alternative delivery media in the form of wireless. This alternative delivery media is selected due to its easy installation and its costefficient infrastructure. MANET is a part of wireless network. It is used for many purposes including the needs for military, disaster recovery, and emergency response. Therefore, an appropriate and reliable routing protocol is required. Selection of a proper routing protocol in emergency broadcast simulation is needed, so that data transmission delivered to each node is well received. Moreover, AODV routing protocol is very suitable for the simulation. It is different from AODV; Reverse-AODV is the development of modified AODV. The use of RAODV routing protocol has been found to be unsatisfactory in emergency broadcast simulation. The number of packet loss is very high and the number of packet received by sample nodes used for research is very low. Optimization is required on this routing protocol, so that the performance of RAODV can be maximized in emergency broadcast simulation.
\end{abstract}

Copy Right, IJAR, 2019, All rights reserved.

\section{Introduction:-}

Infrastructure development that aims to exchange information is intensively carried out nowadays. The equity is almost done in every area. Reliable network is required in order to ease the connection among people. Therefore, infrastructure is needed to be able to connect.

Network infrastructure is divided into several parts. The network infrastructure for transmitting media is divided into wired and wireless network. Currently, wired network is used a lot as a media. It is used until today due to its mobility. However, its infrastructure and maintenance still become a problem because they require a lot of resources. Different from the wired network, today, wireless network is often preferred. It is used a lot for end device communication. Current portable devices such as smart phone, computer, and laptop are equipped with wireless network for communication.

Wireless network used for communication with BTS is a wireless network requiring infrastructure. Different from wireless infrastructure, MANET network is used in several conditions; needs for military, health, emergency communication, and disaster recovery. MANET is used with communication between end devices to the end device without infrastructure assistance.

Corresponding Author:-M. Akbar Marwan.

Address:-Faculty Of Industrial Technology, Gunadarma University. 
Ad-hoc mobile network is a dynamic self-organizing network without any central administrator or infrastructure assistance. If two nodes are not within the transmission range, another node is required to be a connecting router between the two nodes. Mobile device even operate autonomously and communicate through dynamic network. Consequently, the frequent change of network topology is the biggest challenge for many major issues such as routing protocol resilience and performance degradation resilience. However, Proactive routing protocol requires nodes to exchange routing information regularly and calculate the route continuously between any nodes in the network, regardless whether or not the route is used. This means that a lot of network resources such as energy and bandwidth that might not needed by MANET, in which the resources are restricted, are wasted. On the other hand, on-demand routing protocol does not regularly exchange the information. Yet, they find route if it is needed for communication between two nodes. Due to dynamic change in ad-hoc network, the connection between nodes is not permanent. Sometimes, a node cannot send packets to next hop node and in result, the packet may be lost. The packet loss may affect the route performance in different ways. One of which, the loss of the route reply brings more problems because source node must restart the procedure of route discovery.

The disadvantage of existing on-demand routing protocol is their primary route search mechanisms that do not pay much attention to the loss of route reply message. More specifically, most of on-demand routing is currently based on a single route reply. Loss of route reply message may cause significant performance wastage. One of these problems is in the Reverse-AODV routing protocol. On emergency broadcast simulation, the use of RAODV routing protocol may result in the packet loss in an enormous number. Therefore, it is required a development to obtain optimal result for RAODV routing protocol.

\section{Literature Review}

On ad-hoc mobile network, the node may move from one place to another place in various speeds. Consequently, topology in the network is continuously changing and unpredictable. Neighbor's nodes may lose communication only in a short period of time, especially when mobility is high. In the on-demand routing protocol, eliminating communication links between nodes may lead to lost/destroyed routes and packet loss. Particularly, the loss of Route Reply (RREP) of AODV protocol may result in major damage to the AODV protocol.

In fact, RREP AODV messages are obtained at the cost of flooding the entire network or part of the area. Loss of RREP causes source nodes to restart route discovery processes that lead to routing performance decrease such as high power consumption, long end-to-end delays and low packet delivery rates. Therefore, we are considering how to reduce the loss of RREP messages. The use of Reverse-AODV routing protocol in emergency broadcast simulation that is obtained based on diagram using the gnuplot application finds that packet loss on the node sample, node 3 , which can be said that its position is closer to the source /broadcaster, which means there is a very large amount of packet loss, even when it is compared to the received packet, which is only 1/10 of packet loss. Indeed, the routing protocol is incompatible with the simulation emergency broadcast created.

\section{Previous Work}

The use of the first routing protocol is AODV. The use of AODV in the emergency broadcast simulation shows good results. The amount of packet received is very good and the number of packet loss is low. There is no packet loss at all at the nearest node. Then at node 3, packet loss occurs, but in a relatively small number. This is proven in Figure 1. Figure 1 is a node located close to the transmission source (broadcaster). 


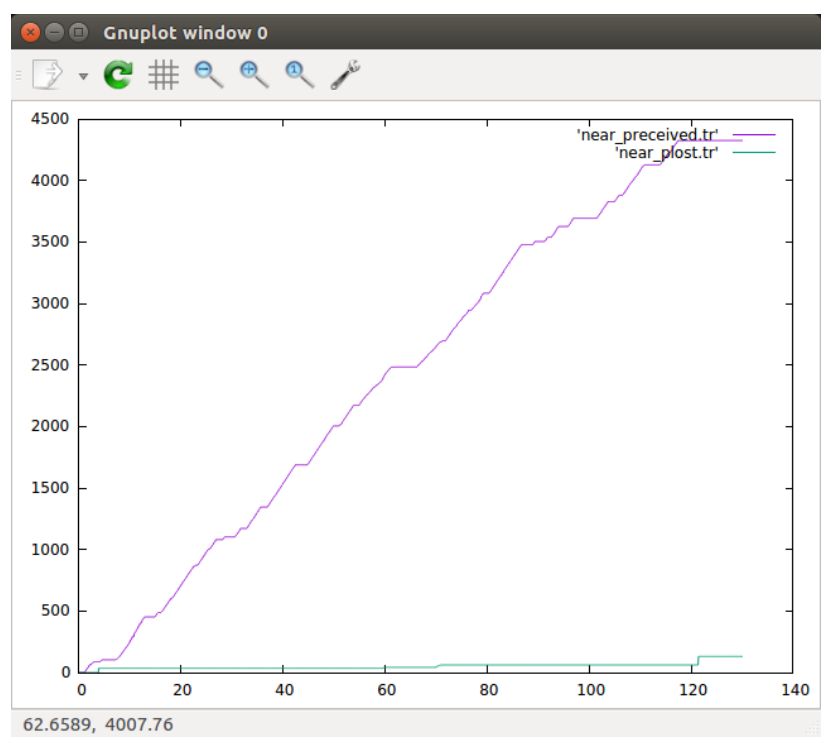

Figure 1:- Packet loss and packet received using AODV routing protocol in node 3

$$
\mathrm{X}=\text { time }
$$

$\mathrm{Y}=$ packet loss/received quantity

The result of the use of AODV routing protocol is shown in Figure 1, the number of packet loss is low, which is around 150. It is accumulated from the time of simulation for 120 minutes. This good result is found in packet received as shown by purple line/curve. In contrast, RAODV routing protocol finds a big number of packet loss, yet small number of packet received. It can be seen in the figure 2 .

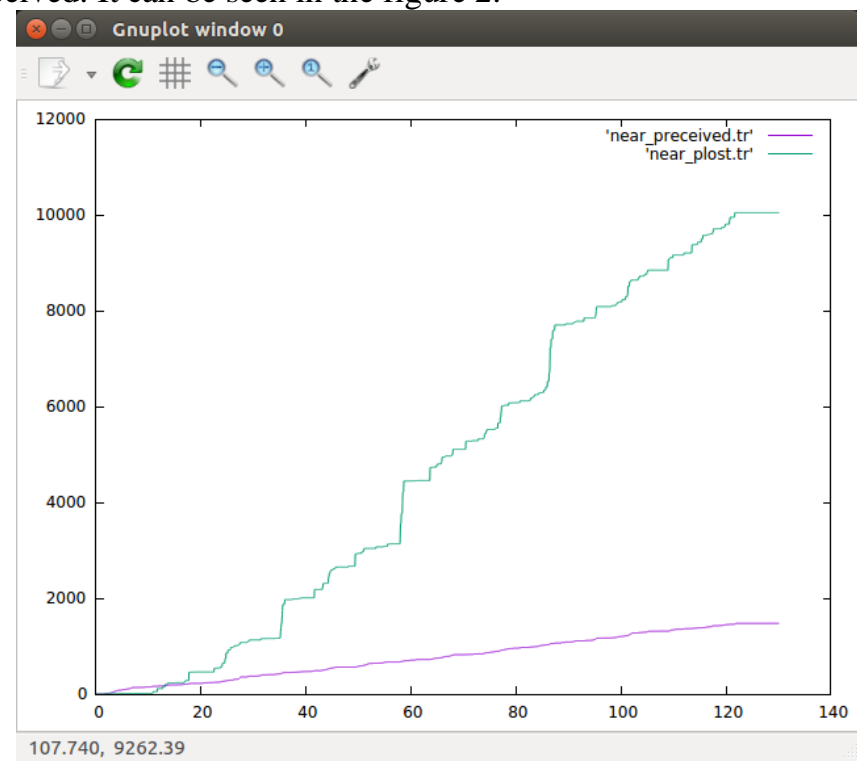

Figure 2:- Packet loss and packet received using RAODV routing protocol in node 3

$$
X=\text { time }
$$

$\mathrm{Y}=$ packet loss/received quantity

Table I:-Comparison On The Use Of Aodv And Raodv Routing Protocol In Node 3

\begin{tabular}{|c|c|c|c|}
\hline Packet/RP & AODV & RAODV & Remark \\
\hline Received & 4500 & 1500 & Decrease $300 \%$ \\
\hline Loss & 150 & 10000 & Increase $6600 \%$ \\
\hline
\end{tabular}

It is found that the number of packet loss is 66 times higher, even packet received on the AODV routing protocol is decreased by $300 \%$. The quantity of packet loss in the simulation using this RAODV routing protocol results in 
higher transmitted packet than using AODV, if it is added to the number of packet received. This is the advantage of RAODV, the data packets transmitted is higher compared to the total number on the AODV routing protocol.

The same thing occurs to the next sample node, which is node 10 . Node 10 is located quite far from the broadcast source and the result can be seen in the figure 3 and 4 .

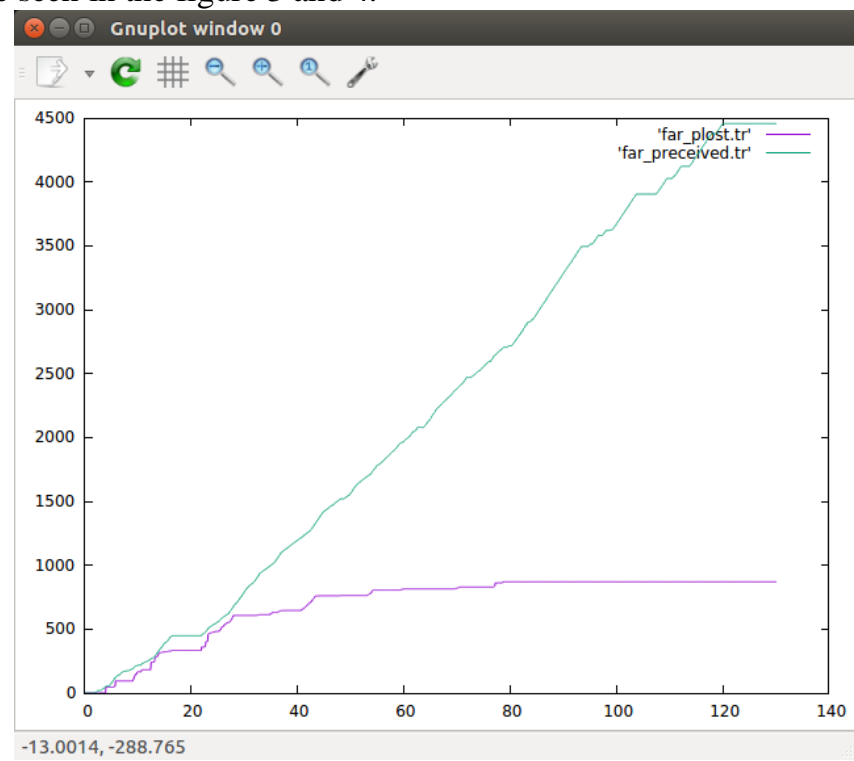

Figure 3:- Packet loss and packet received in node 10 (far) using AODV

$$
\mathrm{X}=\text { time }
$$

$\mathrm{Y}=$ packet loss/received quantity

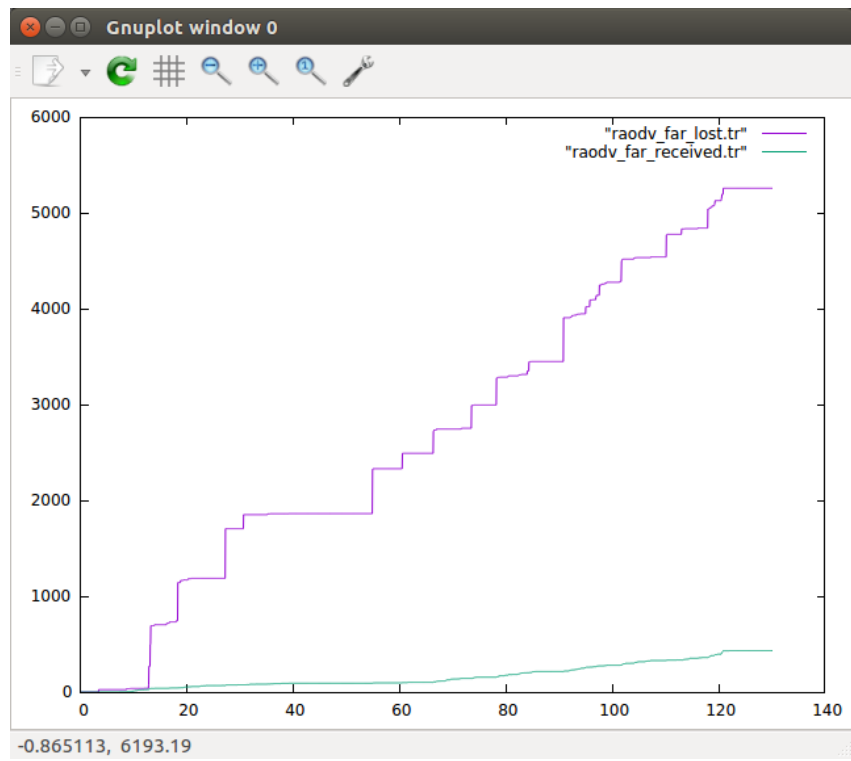

Figure 4:- Packet loss and packet received in node 10 (far) using RAODV

$$
\mathrm{X}=\text { time }
$$

$$
\mathrm{Y}=\text { packet loss/received quantity }
$$

The conclusion is in the following table 2 .

Table Ii:-Comparison On The Use Of Aodv And Raodv Routing Protocol In Node 10 (Far)

\begin{tabular}{|c|c|c|c|}
\hline Packet/RP & AODV & RAODV & Remark \\
\hline Received & 4500 & 480 & Decrease $900 \%$ \\
\hline Loss & 900 & 5250 & Increase $600 \%$ \\
\hline
\end{tabular}




\section{Methods:-}

\section{Proposing The Optimization of Reverse-AODV (ORAODV)}

Analyzing the previous protocol, it can be argued that most on-demand routing protocol, with the exception of routing multipath, use a single route back along the first reverse path to create a routing path. As previously mentioned, with a high mobility, the predetermined turning point can be disconnected and the route reply message from the destination to the source cannot be forwarded. In this broadcast emergency simulation, it can be said that RAODV is not reliable in the transmission from the source to the destination. As it will be explained below

\section{ORAODV Routing Protocol Review}

Due to the large number of packet loss, it needs at least a decrease in the number. Therefore, we need an optimization of RAODV routing protocol for broadcast emergency simulation. In the AODV routing protocol, the route reply from the destination to the source may be disconnected. In this simulation, the cause of the disconnection of the packet is the non-activation of local repair function. This causes the large number of packet loss. Consequently, Optimization of Reverse-AODV (ORAODV) is proposed to optimize RAODV. The explanation on this RAODV modification that becomes one of packet loss number reducer will be explained below.

\section{Path Search in RAODV}

Since RAODV is a reactive routing protocol, there is no permanent route saved in the node. The source node initiates route search procedure by broadcasting. RREQ message carries information as follow (Figure 4).

\begin{tabular}{|c|c|c|}
\hline Type & Reserved & Hop Count \\
\hline Broadcast ID \\
\hline Destination IP address \\
\hline Destination Sequence Number \\
\hline Source IP address \\
\hline Source Sequence number \\
\hline Request Time \\
\hline
\end{tabular}

Figure 5:- Route Request (RREQ) Message in AODV

When the source node concerns a new RREQ message, the number of the broadcast ID increases by 1 . Similarly, the source address and destination address, as well as the broadcast-ID, identifies the unique code of the RREQ packet. The source node broadcasts the RREQ message to all existing nodes within the neighboring Node transmission range. This neighbor node will forward the RREQ to another node in the same way. Because RREQ is broadcasted across the entire network, some nodes may receive multiple copies of the same RREQ. When an intermediary node receives RREQ, the node checks whether it has received RREQ with the same source id and broadcast address. The node gets the first broadcast ID and source address then drops the excessive RREQ messages. The procedure is the same as in RODQ AODV.

When the destination node receives the first route request message, it generates a message called reverse request (RRREQ) and broadcasts it to all neighboring nodes within the transmission range as in the RREQ done by the source node. R-RREQ message carries information as in Figure6. On the other hand, When R-RREQ broadcast message arrives at the intermediary node, its redundancy would be checked. If the message is the same (R-RREQ) message, it will be dropped; otherwise it will be forwarded to all nodes.

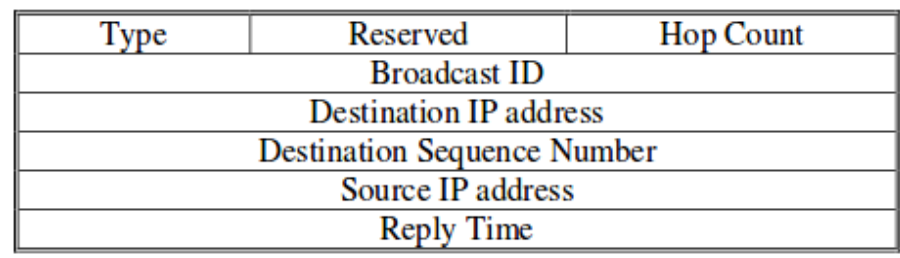

Figure 6:- R-RREQ) Message Format

\section{Optimization of Reverse-AODV}

In the last paragraph of the previous sub-chapter, the cause of the large number of packet loss can be known from the broadcast emergency simulation. Reverse-AODV drops the packet if it finds a similar message. The potential of 
packet loss is massive from the second delivery and so on. In this case, a routing protocol modification is performed to get around the problem. It is impossible to eliminate packet loss, but the quantity of the packet loss itself can be reduced.

Optimization of Reverse-AODV or abbreviated OR-AODV is a routing protocol that aims to reduce the quantity of packet loss. Modifications are made to the oraodv.cc file without changing the header file. Reactivation of local repair function is sufficient to reduce the quantity of packet loss. Local repair itself functions to repair nodes located near the source node.

\section{Result:-}

This chapter will explain the comparison of each node; the sample of this paper is nodes located near and far from the broadcaster, 1 each. Node 3 (near) is chosen because there is packet loss in AODV and there is no packet loss in the other nearby nodes. Figure 7 shows the comparison graphic between RAODV and ORAODV routing protocols in node 3 (near).

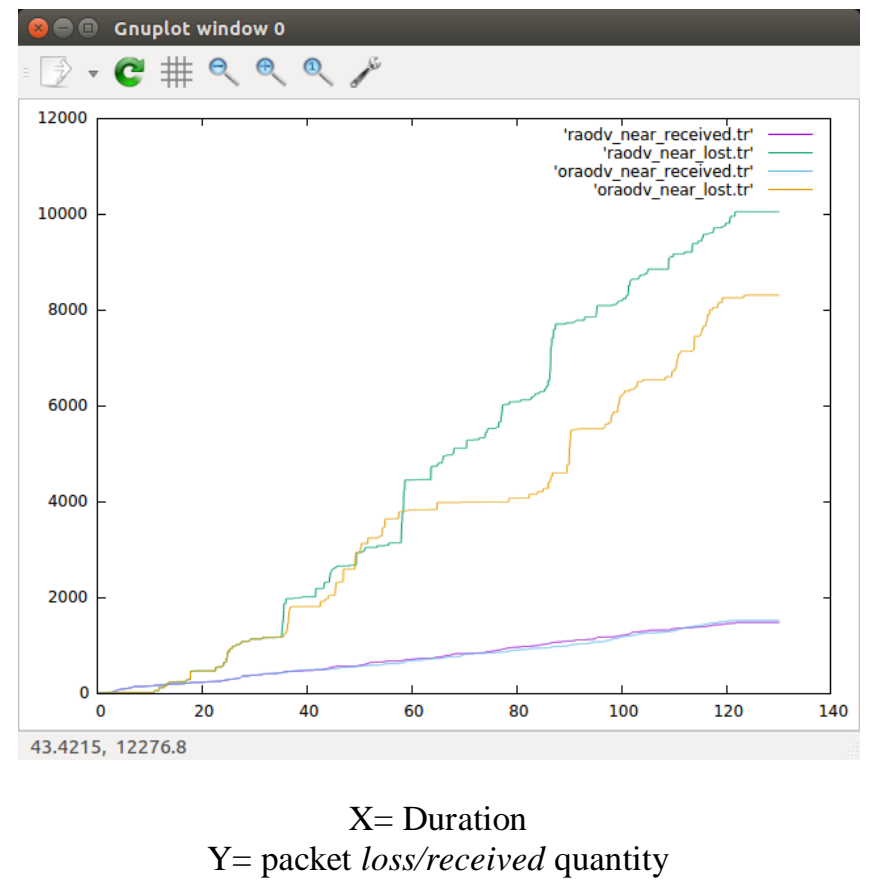

Figure 7:- Comparison graphic between packet loss and received in node 3 between RAODV and ORAODV routing protocols

There is a decrease in the number of packet loss in both RAODV and OR-AODV routing protocols in Figure 7. Based on the graph in Figure 7, RAODV routing protocol has a massive packet loss. There is a decrease in the result of RAODV optimization, OR-AODV, showed by yellow curve/line. Furthermore, a comparison chart between the two routing protocols in a far node, node 10 in this simulation, will be presented. 


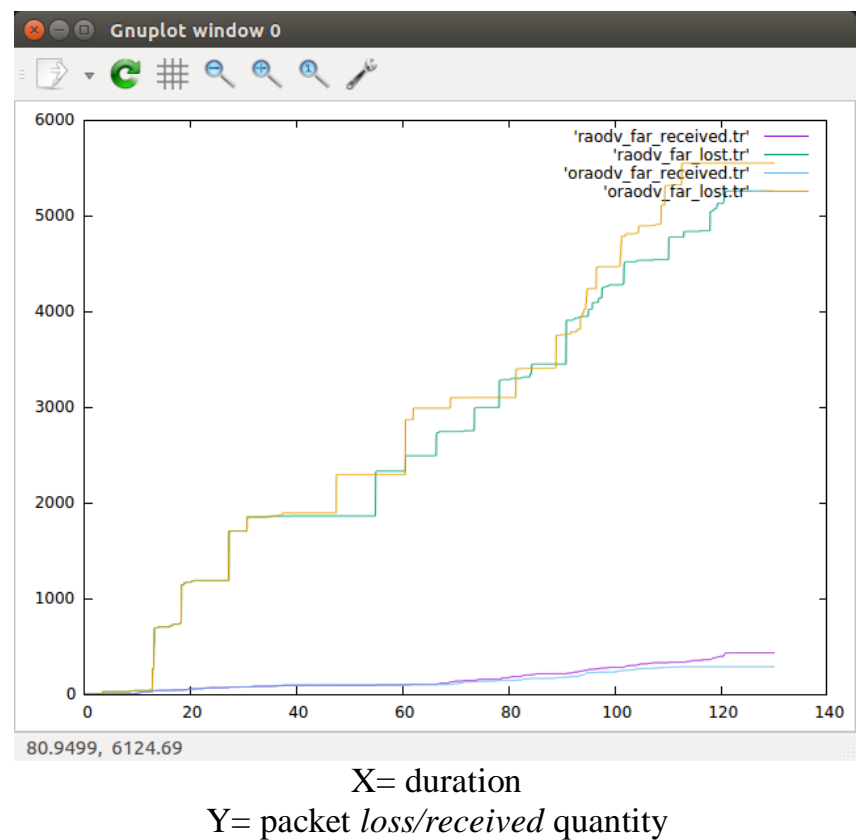

Figure 8:- Comparison graphic of packet loss and received in node 10 between RAODV and ORAODV routing protocols

In contrast to the near node, the far node experiences an increase in packet loss quantity and a decrease in packet received quantity, the decrease in packet received is approximately $21 \%$ and the increase in packet loss is $5.4 \%$. It can be said that there is no significant increase in packet loss and decrease in packet received in terms of quantity, but the number of percentage exceeds the decrease of packet loss quantity in node 3 (near). The explanation of the packet loss and packet received quantity in broadcast emergency simulation along with its percentage is shown in Table 3 and Table 4.

Table Iii:-Table Of Quantity Comparison In Node 3 (Near)

\begin{tabular}{|c|c|c|c|}
\hline Packet/RP & RAODV & ORAODV & Percentage \\
\hline loss & 10000 & 8300 & Reduce $17 \%$ \\
\hline received & 1500 & 1500 & Constant \\
\hline
\end{tabular}

Table Iv:-Table Of Quantity Comparison In Node 10 (Far)

\begin{tabular}{|c|c|c|c|}
\hline Packet/RP & RAODV & ORAODV & Percentage \\
\hline loss & 5.250 & 5550 & Increase 5,4\% \\
\hline received & 480 & 380 & Decrease $21 \%$ \\
\hline
\end{tabular}

\section{Conclusion:-}

It can be concluded that the RAODV routing protocol optimization is successful. Based on table 1 and table 2, the reduction of packet loss quantity is $17 \%$ in the near node. However, the decrease of packet received quantity in the far node is quite massive in terms of percentage, not in quantity. The massive packet loss in the RAODV routing protocol usage happens when there is a similar R-RREQ message reception in the intermediate node, causing an immediate drop of the packet. Meanwhile, the broadcast is carried out continuously during the simulation in the broadcast emergency simulation, causing a drop in each repetition of packet delivery. 


\section{Reference:-}

1. Bae, J. H., Kim, D-M., Tae-Hyoun Kim, Jaiyong Lee. An AODV-based Efficient Route Re-Acquisition Scheme in Ad Hoc Networks.

2. Bai, R and Singhal, M., "Salvaging Route Reply for On-Demand Routing Protocols in Mobile Ad-Hoc Networks" in MSWIM 205, Montreal, Quebec, Canada. Oct 2005.

3. Kim, Chonggun. Talipov, Elmurod. and Ahn, Byoungchul. "A Reverse AODV Routing Protocol in Ad Hoc Networks", Korea, 2006. pp. $522-531$.

4. Lee, C. K.-L., Lin, X.-H. and Y.-K. Kwok, "A Multipath Ad Hoc Routing Approach to Combat Wireless Link Insecurity". Proc. ICC 2003, vol. 1, pp. 448-452, May 2003.

5. Marina, M. K. and Das, S. R. "On-Demand Multi Path Distance Vector Routing in Ad Hoc Networks," Proc. ICNP 2001, pp. 14- 23, Nov. 2001.

6. Marwan, M. Akbar. "PengembanganAlgoritma Multipath Dominator pada Mobile Ad Hoc Network". Disertasi. Depok. Indonesia 2015.

7. Nasipuri, A and S. R. Das, "On-Demand Multipath Routing for Mobile Ad Hoc Net- works," Proc. ICCN 1999, pp. 64-70, Oct. 1999.

8. NS, The UCB/LBNL/VINT Network Simulator (NS), http://www.isi.edu/nsnam/ns/, 2004.

9. Perkins, C., E. Belding-Royer "Ad hoc on-Demand Distance Vector (AODV) Routing", RFC 3561, July 2003

10. Perkin, C. E. and Royer, E. M. "Ad hoc on-demand distance vector routing," in Proc. WMCSA, New Orleans, LA, Feb. 1999, pp. 90-100. 